\title{
RAFFITTIMENTO GRAVIMETRICO DELLA ZONA CENTRO ORIENTALE DELL'ARCO ALPINO
}

\author{
Armando Norinelli
}

La regione dell'Italia settentrionale, limitata a nord e ad est dal confine di Stato, a sud dal $P_{0}$ e ad ovest approssimativamente dalla linea congiungente Cremona-Desenzano-Tione-Tirano-confine di Stato, venne rilevata nel 1951, con misure gravimetriche a larghe manlie, dal prof. Carlo Morelli ( ${ }^{1}$ ).

La Commissione Geodetica Italiana esprimeva però i voti che la densita delle stazioni gravimetriche da eseguire su tutto il territorio italiano, per la compilazione di una carta gravimetrica, fosse di una stazione ogni $100 \mathrm{~km}^{2}$. L'Istituto di Geodesia e Geofisica dell'Università di Padova si assunse allora l'incarico del raffitimento gravimetrico della succitata regione. trascurando quelle zone in cui successivamente al 1951 vennero raccolte altre osservazioni gravimetriche dall'Osservatorio Geofisico di Trieste $\left({ }^{2}\right)\left({ }^{3}\right)$, dall'Istituto di Geodesia e Geofisica di Padova ( $\left.{ }^{4}\right)$ e dall'Istituto Geografico Militare, il quale esegui alcune stazioni a cavallo del confine di Stato.

Il lavoro da fare rimaneva comunque considerevole. Per la sua attuazione il Prof. Giovanni Silva poté ottenere dal Consiglio Nazionale delle Ricerche $i$ mezzi finanziari occorrenti ed in prestito dalla Soc. Montecatini il gravimetro Western G 4 A, n. 11 di matricola. Il rilievo. iniziato con alcune stazioni a sud del lago di Garda nel mese di agosto del 1953, venne ripreso e concluso nel periodo luglio-agosto 1954 .

Si dà qui relazione di quanto s’è fatto, raccogliendo alla fine i dati osservati e le monografie delle stazioni. Non si è proceduto alle consuete riduzioni, poiché $\mathrm{i}$ relativi calcoli verranno condotti successivamente dall'Istituto di Geodesia e Topografia dell'Università di Pisa, incaricato di tale compito su scala nazionale dalla Commissione Geodetica Italiana. Tuttavia ai valori osservati vennero applicate le riduzioni in aria libera e per la piastra. Benché questa riduzione di Bouguer, che chiamerò semplice col Baeschlin $\left({ }^{5}\right)$, costituisca una prima approssimazione di riduzione della gravità al geoide, il risultato cui 
conduce, e ciò̀ la costruzione della carta delle isoanomale secondo Bouguer, che chiamerò di conseguenza col nome semplici, anche per distinguerle dalle modificate e generalizzate, servirà per studi in corso presso l'Istituto di Geodesia e Geofisica di Padova, tendenti ad avere buone indicazioni sullammontare delle correzioni nelle livellazioni di alta precisione in causa del non parallelismo delle superficie di livello $\left({ }^{6}\right)$.

Il rilievo venne effettuato per polignoni chiusi, partendo la mattina e ritornando la sera alla medesima stazione di riferimento. Le stazioni non vennero, per ragioni di economia, ribattute una seconda volta. come sareble stato desiderabile, per avere un controllo delle osservazioni ed una maggoiore precisione.

Data l'orografia accidentata della regione, le altitudini delle stazioni programmate presentavano lungo i vari profili notevoli salti: e poiché d'altra parte il campo della vite di lettura del gravimetro usato era di circa 100 mgal. si ritenne opportuno di sperimentare due metodi di rilievo:

a) passare direttamente da una stazione alla contigua, assoggrettando il gravimetro a frequenti cambi di campo:

b) oppure ripetere lo stesso poligono, fermandosi ogni volta solo in quelle stazioni, nelle quali la misura rientrava nel campo del gravimetro.

Il secondo metodo riuscì più vantagrgioso del primo e venne perciò seguito quando possibile.

Le stazioni di cui si riportano i dati sono 181; ma, a causa degli inevitahili cambi di campo fatti durante il tragitto nel passaggio da qualcuna di esse alla successiva, risultano in effetti più numerose.

Strumento -- Dello strumento Western suddetto venne fatta una indagine accurata degli errori progressivi della vite di lettura (7) ed eseguita la taratura sulla base Bologna-Ferrara $\%$ prima e dopo la campagna gravimetrica.

La prima taratura, di cui venne dato conto in un precedente lavoro $\left({ }^{4}\right)$, portò per la costante di scala al valore $-0,08512+0,00003$ : la seconda taratura, eseguita nei gioni 4 e 5 novembre 1954 , ripetendo ciascun tronco della base tre volte, diede quale valore della costante di scala $-0,08513 \div 0,00007$, valore ottenuto dai dati di taratura col metodo delle osservazioni mediate, analogamente a quanto fatto la prima volta. 
Per la verifica della compensazione barometrica dello strumento ci si accontentò del buon accordo che presentano le osservazioni eseguite dallo scrivente e quelle eseguite nello stesso posto dal prof. Morelli con gravimetro Worden, le cui misure sono indipendenti dalle variazioni della pressione atmosferica.

Stazioni di riferimento - Per la zona a sud del Lago di Garda venne scelta, quale stazione di riferimento, Verona [N. VIlI del rilievo gravimetrico precedentemente eseguito dall'Istituto $\left.\left({ }^{4}\right)\right]$, con $g_{\text {oss }}$ $=980,66387$ gal. Tale valore venne determinato collegando la stazione direttamente con Padova, dove si è assunto al piano terreno dell'Osservatorio Astronomico (Sala di gravità G. Lorenzoni) 980,6575 gal, che è il valore di rijerimento della gravità per tutto il rilievo.

Poiché la stazione di Padova della rete gravimetrica fondamentale italiana $\left({ }^{9}\right)$ venne stabilita a $\mathrm{m} 4,15$ esternamente ed in asse al cancello d'ingresso al giardino dell'Osservatorio astronomico, venne ad essa accuratamente collegata la stazione gravimetrica della Sala G. Lorenzoni: risultò all'esterno del suddetto cancello esterno dell'Osservatorio astronomico un valore della gravità di 0,11 mgal maggiore di quello della Sala G. Lorenzoni.

Tenendo conto delle differenze di gravità comunicatemi dal dottor Cunietti, i valori della gravità nelle seguenti stazioni della rete fondamentale risultarono: 980,61050 a Trento (di fianco alla porta Aquila dalla parte di via Ventuno presso il caposaldo orizzontale dell' I.G.M.); 980.57469 a Bolzano (Cimitero, in asse al cancello di sinistra guardando il cimitero stesso); 980,62948 a Primolano (in Piazza S. Rocco in asse alla fontanella sita all'angolo di detta piazza); 980,66895 a Cervignano (in prossimità del ponte sul torrente Aussa, nella piazzuola a fianco del distributore di benzina presso il palo terminale della linea elettrica, al quadrivio Udine-CervignanoTrieste-Venezia).

Quali stazioni di riferimento della zona montana vennero prescelte: Trento, Bolzano della rete fondamentale; Spondigna (n. 648 del presente rilievo) e Brunico (n. 671), collegate direttamente con Bolzano; Alleghe (n. 633) e Tolmezzo (n. 635) il cui valore della gravità venne determinato nel modo seguente:

vennero collegate due volte tra loro Alleghe e Belluno (n. 634) ed in un secondo tempo Belluno e Primolano (due volte), stazione principale della rete fondamentale; poi si collegò Alleghe con Tolmezzo (una voltal e questa con Cervignano (due volte), stazione inter- 
media della rete fondamentale. L'errore di chiusura della spezzata Primolano-Belluno-Alleghe.Tolmezzo, Cervignano risultò $+0.04 \mathrm{mgal}$.

La compensazione venne fatta col metodo dei minimi quadrati, considerando i dati della Commissione Geodetica Italiana come imposti ed invariabili ed attribuendo quali pesi alle differenze di gravità osservate numeri proporzionali direttamente al numero dei collegamenti fatti ed inversamente alla durata del percorso. Infatti, poiché la deriva durante la campagna non risultò regolare come in laboratorio (di ciò fu data già relazione in ( $\left.{ }^{4}\right)$ ), è da ritenere tale irregolarità in relazione anche con la durata del tragitto.

Riporto a fianco dei singoli tratti le differenze $J g$ aspresin in mgal) della gravita osservate. i tempi $t$ di percorso ed i pesi $p$ corrispondenti.

$\begin{array}{lccc}\text { Primolano-Belluno } & \lrcorner g=-23,06 & t=1^{\mathrm{h}} & p=13 \\ \text { Belluno-Alleghe } & -148,47 & 2 & 6,5 \\ \text { Alleghe-Tolmezzo } & +159,40 & 6,5 & 1 \\ \text { Tohnezzo-Cervignano } & +51,64 & 1,5 & 8,7 \\ \text { I valori compensati sono riportati nella tabella lI. } & \end{array}$

Precisione dei dati osservati -- Delle misure gravimetriche eseguite nella regione euganeo-berico-lessinea ottenni quale errore medio $\pm 0,06 \mathrm{mgal}$.

Mancando qui la possibilita di ricavare un analogo errore medio e considerando che le condizioni di lavoro sono state assai meno buone, ho ritenuto di attribuire ai valori osservati nel presente rilievo una incertezza di 2 decimi di mgal.

A stabilire tale criterio hanno valso anche ventidue confronti tra i valori osservati dal prof. Morelli e da me nei medesimi posti.

Nelle tabelle I e II le stazioni si succedono in ordine cronologico. Il numero di partenza è il 542 , in continuazione col precedente rilievo dell'Istituto $\left(^{*}\right)$.

Nella tabella I vengono riportati il numero d'ordine della stazione, il nome della località ed un succinta monografia: ulteriori dcttagli, o la fotografia, possono essere richiesti all'Istituto.

Come riferimento altimetrico vennero cercati caposaldi orizzontali (cs. o.) o verticali (cs. v.) di livellazione geometrica dell'Istituto 
Geografico Militare, in mancanza dei quali ci si appoggriò al pianc del ferru (p. d. f.) delle linee ferroviarie o a punti trigonometrici (p. t.) Quando questi ultimi risultarono deteriorati o rifatti, ci si riferì alla quota riportata dalle carte al 25.000 e per precisare tali casi si è usata l'espressione punti trigonometrici suolo (p.t.s.). Si è fatto ricorso anche a punti quotati(p. q.) sulle carte al 25.000 e solo una volta alle curve di livello (c. l.).

Per le stazioni del prof. Morelli (M.) ripetute in questo rilievo, venne adottata la quota riportata nella sua publblicazione ( ${ }^{1}$ ).

Uteriori abbreviazioni usate nella tab. I sono: C. P. $=$ Chiesa parrocchiale e S. F. = stazione ferroviaria.

Yella tal,. Il compaiono: il numero d'ordine della stazione: la quota $h$; il valore della gravità osservata $g$, corretto per la deriva dello strumento e per la variazione diurna della gravità terrestre: le coordinate geografiche riferite all'ellissoide internazionale orientato a Monte Mario: latitudine $\uparrow$ e longitudine $\lambda$, contata ad ovest rispetto al meridiano di M. Mario, tranne quand'è seguita da " $\mathrm{E}$ ); la gravità normale $\gamma_{0}$. calcolata secondo la formula internazionale; la riduzione in aria libera $\delta g$. ottenuta moltiplicando la quota per 0.30855 ; la riduzione per la piastra $\delta^{\prime} g$, calcolata con la formula: ${ }^{\prime} g=-0,04191$ $D h$, dove in $I$ '̇ stato introdotto il valore della densita media del terreno sino al livello del mare, ricavata dalla "Carta della densità media ") del prof. Vecchia $\left({ }^{10}\right)$; la gravita $g^{\prime}$, ridotta per la quota e per la piastra; la anomalia di Faye $g+\bar{c} g-\gamma_{0}$ e quella semplice di Bouguer $g_{0}^{\prime}-\gamma_{0}$. I valori della gravità e delle correzioni sono espressi in millesimi di gal.

La carta allegata riporta la distribuzione delle stazioni eseguite nell attuale raffitimento. Ne compaiono anche del rilievo gravimetrico precedente $\left({ }^{4}\right)$ con la densità di circa 1 per ogni $100 \mathrm{~km}^{2}$; figurano inoltre stazioni del prof. Morelli $\left({ }^{1}\right)\left({ }^{2}\right)\left({ }^{3}\right)$ e del dott. Cunictti $\left({ }^{11}\right), \mathrm{i}$ cui dati hanno servito al tracciamento delle isoanomale semplici secondo Bouguer con l'equidistanza di $10 \mathrm{mgal}$. linec tracciate appositamente per lo scopo dichiarato all inizio.

Padova - Istituto di Geodesia e Geofisica - Marzo 1955.

\section{RI.ASSUNTO}

Vengono raccolti 181 dati osservati con un gravimetro Testern dall Istituto di Geodesia e Geofisica dell Università di Padova nella regione citata nel titolo e nel territorio a sud del Lago di Garda. 
$\grave{E}$ unita una carta delle isomomale secondo Bouguer (semplici), utile per un'applicazione della gravimetria alla livellazione.

\section{SUMMARY}

181 observations tahen with a IT estern Gravimeter by the Institute of Geodesics and Geophysics of the University of Padova in the region cited in the title, and in the territory south of the Lake of Garda. have been collected.

A map of the isoanomals (simple) according to Bouguer, useful for the application of gravimetry to level measurements, is attached.

\section{BIBLIOGR AFIA}

(1) C. Morell, Rilievo gravimetrico e riduzione isostatica nellItalia nordorientale. Tecnica Italiana, Trieste, anno VI, n.. 3, 4, 1951 (Pubblicaz. n. 6 dell'Istituto di Geodesia e Geofisica dell'Università di Padova = I.G.G.U.P.).

(2) C. Morelli, Rilievo geofisico dell'Altipiano di Asiago. Annali di Geofisica, vol. VI, n. 2, 1953.

(3) C. Morelti, Rilievo gravimetrico e magnetico del Veneto centro.orientale. Metano, anno VIII, n. 12, 1954.

(t) A. Norikelli. Nuovi dati geofisici sul distretto eruttivo euganeo-bericolessineo. Parte I: Dati gravimetrici. Bollettino di Geodesia e Scienze affini, vol. XIV.

n. 1, 1955 (Pubblicaz. n. 18 dell'I.G.G.U.P.).

(5) C. F. Baeschlin, Lehrbuch der Geodasie. Zürich, Orell Füssli, 19.48, pag. 691.

(b) G. SiLva, Sull'uso attuale della gravimetria in sussidio della livellazione. Parte I e II. Rendiconti Accademia dei Lincei, serie VIII, vol. XVI, fasc. 2 e 3. 1954 (Pubblicaz. n. 13 dell'I.G.G.U.P.).

(7) A. Norivelul, Sugli errori progressivi delle letture di un gravimetro. Annali di Geofisica, vol. VIII, n. 2, 1955 (Pubblicaz. n. 17 dell'I.G.G.U.P.).

(8) C. Monelli, Primo contributo per una rete gravimetrica fondamentale in Italia. Annali di Geofisica, vol. V, n. 1, 1952.

(9) M. Cunietti e G. Inghilleri, La rete gravimetrica fondamentale italiana. Pubbl. della Commissione Geodetica Italiana, 1955, in corso di stampa.

(10) O. Veсchis, Carta della densità sino al livello del mare nell'talia settentrionale. Bollettino di Geodesia e Scienze Affini, anno XI. n. 3, 1952.

(11) M. CusietTI, 51 Misure di gravità relativa eseguite in Italia settentrionale nel 19.49. Rivista Geomineraria, anno X, n. 2, 1949. 
TABLLLA I - Monografia delle stazioni gravimetriche (*)

\begin{tabular}{|c|c|c|c|}
\hline $\begin{array}{l}\text { N. } \\
1\end{array}$ & $\begin{array}{l}\text { Locilità } \\
\qquad 2 \\
\end{array}$ & $\begin{array}{c}\text { Monografic } \\
3\end{array}$ & $\begin{array}{c}\text { Riferimento } \\
\text { altimetrico } \\
4\end{array}$ \\
\hline $5+2$ & Bosco di Zezio & $\begin{array}{l}\text { C. P.: vicino allo spigolo N. O. del cam- } \\
\text { panile }\end{array}$ & p. t. \\
\hline $5-13$ & Cere: & Scuole comunali: al centro dellatrio & M. \\
\hline $5+-1$ & Bovolone & S. F.: a m 2 dallentrata & p. d. E. \\
\hline 545 & Isola dellal Scialia & Oratorio parr.: in centro alla stanza n. 7 & M. \\
\hline $5+6$ & Buttapictra & S. F.: a m 3 dallentratal & p. d. f. \\
\hline 547 & Vigasio & S. F.: a m 2 dall'entrata & p. d. f. \\
\hline $5+8$ & Villafranca & S. F.: a m 6 dalla porta principale & p. d. f. \\
\hline 5.49 & Dossobuono & S. F.: tra le due colonne centrali & p. d. f. \\
\hline 550 & Mozzecane & S. F.: tral le due colonne centrali & p. d. f. \\
\hline 551 & Pozzolo & S. F.: presso lo spigolo N. O. & M. \\
\hline 552 & Marmirolo & S. F.: a m 9 dall'entrata & p. d. E. \\
\hline 553 & Mantoval & $\begin{array}{l}\text { Ginnasio-Liceo in ria Pompanazzo: davanti } \\
\text { al portone }\end{array}$ & M. \\
\hline 554 & Castelbelforte & C. P.: a m 5 dalla portal principale & p. t. \\
\hline 555 & Nogara & $\begin{array}{l}\text { S. F.: a } m+\text { dalla finestra destra, guardan- } \\
\text { do, della facciata } S \text {. O. }\end{array}$ & p. d. f. \\
\hline 556 & Maccacari & C. P.: davanti al campanile & p. t. \\
\hline 557 & Casaleone & $\begin{array}{l}\text { S. F.: a m } 3 \text { dalla porta destra. guirdando. } \\
\text { della facciata N. O. }\end{array}$ & p. d. f. \\
\hline 558 & Ostiglia & S. F.: davanti alla porta centrale & M. \\
\hline 559 & Rergantino & C. P.: vicino alla parete ovest del campanile & p. t. \\
\hline 560 & Nosedole & C. P.: a m 2 dalla porta principale & p. t. \\
\hline 561 & Barbasso & Canonical: a $m+$ dall'entratta & p. t. \\
\hline 562 & Borgoforte & S. F.: accinto allo spigolo S. O. & p. d. f. \\
\hline 563 & Sommacampagna & S. F.: a in 6 dalla porta centrale & p. d. f. \\
\hline 564 & Castelnuovo & S. F.: a m 3 dalla porta centrale & M. \\
\hline 565 & Sirmione & $\begin{array}{l}\text { Chicsa S. Pietro: a } m+\text { dalla parete sud } \\
\text { del campanile }\end{array}$ & p. t. \\
\hline 566 & $\begin{array}{l}\text { Valeggio sul Min- } \\
\text { cio }\end{array}$ & S. F.: tra la stazione ed il binario & p. d. E. \\
\hline 567 & Costermano & Stazione tram: di fronte, a $\mathrm{m} 2$ dalle rotaic & p. d. E. \\
\hline 568 & Peschiera & $\begin{array}{l}\text { C. P.: a } \mathrm{m} 2 \text { dalla parete nord in corr. } \\
\text { del cs. }\end{array}$ & cs. $v$. \\
\hline 569 & Bardolino & Chiesa di S. Severo: al m 3 dalla porta cieca & cs. $v$. \\
\hline 570 & $\begin{array}{l}\text { S. Martino della } \\
\text { Batt:aglia }\end{array}$ & S. F.: a $\mathrm{m} 3$ dalla porta principale & p. d. f. \\
\hline 571 & Desenzano & C. P.: davanti allia porta principale & Cunictti \\
\hline 572 & Montichiari & $\begin{array}{l}\text { Chiesa s. Pancrazio: a } m 6,5 \text { dalla parete } \\
\text { est del campanile }\end{array}$ & p. t. \\
\hline 573 & $\begin{array}{l}\text { Castiglione delle } \\
\text { Stiviere }\end{array}$ & C. P.: a m 18 dal portale destro guardando & p. t. \\
\hline 574 & Sacca & Corte Molinara: a $m+$ dalla porta $n .7$ & p. t. \\
\hline 575 & Castellucchio & S. F.: a $\mathrm{m} 3$ dalla porta centrale & p. d. f. \\
\hline 576 & Torre de' Picenardi & S. F.: a $\mathrm{m} 3$ dalla porta centrale & p. d. f. \\
\hline 577 & Assola & S. F.: davanti alla porta centrale & p. d. t. \\
\hline 578 & $\underset{\text { ra }}{\text { Mariana Mantova- }}$ & Piazza Castello: a $m+$ dalla fontana & p. t. \\
\hline 579 & Ceresara & $\begin{array}{l}\text { Piazza Cistello: a m } 3 \text { dalla Torre e a } \\
\text { m 12 dal Municipio }\end{array}$ & p. t. \\
\hline
\end{tabular}

(*) Per le abbreviazioni redasi testo. 


\begin{tabular}{|c|c|c|c|}
\hline $\begin{array}{c}\mathrm{N} . \\
1\end{array}$ & $\begin{array}{l}\text { Localirì } \\
\qquad 2\end{array}$ & $\begin{array}{c}\text { Monogratic } \\
3\end{array}$ & $\begin{array}{l}\text { Riferimento } \\
\text { altimetrico } \\
4\end{array}$ \\
\hline 58010 & Nedole & Scuole comunali: all'inizio della scalinata & p. i. \\
\hline 581 & Acquatredda & C. P.: a $m+$ dallia porta principale & p. t. \\
\hline $5 \times 2$ & Giazuolo & C. P.: a $\mathrm{m} 13$ dalla porta centrale & is. 0. \\
\hline 583 & $\begin{array}{l}\text { S. Matteo delle } \\
\text { Chiaviche }\end{array}$ & C. P.: allinizio dellat scalinata & p. 4 . \\
\hline 584 & Sabbioneta & C. P.: a m 2.5 dillal porta & p. t. \\
\hline 585 & Gussola & C. P.: a $\mathrm{m}$ ls dalla portal del companile & p. t. \\
\hline 586 & $\begin{array}{l}\text { S. Giovanni in } \\
\text { Croxe }\end{array}$ & S. F.: a m 3 dall entratia & n. d. 1 \\
\hline 587 & Calliano & S. F.: a m 3 dalla porta centrale & p. d. f. \\
\hline 588 & Mattarello & S. F.: davinti allentrata & p. d. f. \\
\hline 589 & Rovereto & $\begin{array}{l}\text { S. F.: davanti all arco centrale del porticitus } \\
\text { dingresso }\end{array}$ & M. \\
\hline 590 & Serravalle & S. F.: a m 2 dallentrata & p. a. i. \\
\hline 591 & Ala & S. F.: a in 3 dalla porta centrale & p. d. f. \\
\hline 592 & $\begin{array}{l}\text { Borghetto sull:Adi- } \\
\text { ge }\end{array}$ & S. F.: a m 2 dallentrata & p. a. 1. \\
\hline 593 & Ponte Nova & $\begin{array}{l}\text { Ponte a } \mathrm{km} 1.7 \text { in linea daria dalla chiesa } \\
\text { di ponte Nova. venendo da Bolzano: a } \\
\text { n } 20 \text { dallestremo destro del parapetto } \\
\text { a valle in allineamento }\end{array}$ & p. q. \\
\hline 594 & Meida & C. P.: davanti all'entrati: & p. q. \\
\hline 595 & Canazei & $\begin{array}{l}\text { Chiesa vecchial a } \mathrm{m} \text { o dalla pirete est del } \\
\text { campanile }\end{array}$ & i. \\
\hline 596 & $\begin{array}{l}\text { Passo di Costalun- } \\
\text { ga }\end{array}$ & $\begin{array}{l}\text { Alhergo al l'asso: di fronte allentratia cen- } \\
\text { trale sul ciglio della strala }\end{array}$ & $\mathrm{M}$. \\
\hline 507 & Preclazzo & S. F.: tra il porticato ed il binario & $M$. \\
\hline 598 & Moena & $\begin{array}{l}\text { Ponte di pietra allingresso del paese venen- } \\
\text { do da Predazzo: a } m \text { li dall'asse del } \\
\text { ponte }\end{array}$ & p. प. \\
\hline 599 & Tesero & S. F.: a m 2 dalla parete cst & p. d. if. \\
\hline 600 & $\begin{array}{l}\text { Castello di Fiem- } \\
\text { me }\end{array}$ & S. F.: fra il porticato ed il binario & M. \\
\hline 601 & Sin Lugano & S. F.: a $\mathrm{m}+$ dalla portil della parete nord & I) d. $\mathrm{f}$. \\
\hline 602 & Arabba & C. I'.: a m 2 dial cancello d'entrata & M. \\
\hline 603 & Colfosio & $\begin{array}{l}\text { C. P.: a m } 1,5 \text { dal cancello in legno del } \\
\text { cimitero }\end{array}$ & p. t. s. \\
\hline $60 t$ & $\begin{array}{l}\text { Plian - Val Gar- } \\
\text { denal }\end{array}$ & $\begin{array}{l}\text { Scalo ferr.: a m } 30 \text { ad ovest dalla rimessi } \\
\text { locomotive e a } \mathrm{m}+\text { a sucl dei binari }\end{array}$ & M. \\
\hline 605 & Ortisei & S. F.: davanti al cancello & M. \\
\hline 606 & Cencenighe & $\begin{array}{l}\text { Ponte sul torrente Biois: a } \mathrm{m} 3 \text { sulla destra. } \\
\text { in allineamento col pirapetto a monte }\end{array}$ & p. $\mathbf{q}$. \\
\hline 607 & Forno di Canale & $\begin{array}{l}\text { C. P.: a m } 2 \text { dal cancullo dalla parte sud } \\
\text { del campanile }\end{array}$ & M. \\
\hline 608 & l'asso Falzarego & $\begin{array}{l}\text { Monumento ai Caduti: a in l dal primo } \\
\text { gradino della scaletta }\end{array}$ & p. $\mathbf{q}$. \\
\hline 609 & Cortina d'Ampezzo & $\begin{array}{l}\text { S. F.: a m l dalla secondia portil d'ingresso } \\
\text { allo scalo merci }\end{array}$ & M. \\
\hline 610 & Ospitile & S. F.: tra la porta centrale e i binari & p. d. f. \\
\hline 611 & Stibiziane & $\begin{array}{l}\text { Villa Gregoriana: a m } 3 \text { dalla facciata tra } \\
\text { le due porte }\end{array}$ & p. 4 . \\
\hline
\end{tabular}




\begin{tabular}{|c|c|c|c|}
\hline $\begin{array}{c}\text { N. } \\
1\end{array}$ & $\begin{array}{l}\text { Localita } \\
2 \\
\end{array}$ & $\begin{array}{c}\text { Monografie } \\
3\end{array}$ & $\begin{array}{l}\text { Riferimento } \\
\text { altimetrico } \\
\qquad 4\end{array}$ \\
\hline 612 & Passo S. Pellegrino & $\begin{array}{l}\text { Ponte sul torrente Marzon sulla statale del- } \\
\text { le Dolomiti al km 150: sulla destra a } \\
\text { m } 4 \text { dal primo paletto in ferro del pari- } \\
\text { petto a valle } \\
\text { Rifugio S. Pellegrino: a m } 3 \text { dalla faciata } \\
\text { sud in allineamento con la est }\end{array}$ & p. q. \\
\hline 614 & Paneveggio & $\begin{array}{l}\text { Ponte sul Rio Juribello: a m } 17 \text { dall'estre- } \\
\text { mo sinistro del parapetto a valle }\end{array}$ & p. q. \\
\hline 615 & S. Martinu di C. & C. P.: a m 2 dalla porta principale & p. q. \\
\hline 616 & Castello Tesino & $\begin{array}{l}\text { Al km } 34,800 \text { sulla strada Castel Tesino- } \\
\text { Passe Broccon: accanto al cippo km } 34,800\end{array}$ & c. $\mathrm{l}$. \\
\hline 617 & Passo Broccone & $\begin{array}{l}\text { Monumento ai Caduti: a } m 5 \text { dal primo } \\
\text { gradino della scaletta }\end{array}$ & p. q. \\
\hline 618 & Prade & C. P.: a m 3 dalla porta laterale & M. \\
\hline 619 & Mezzano & C. P.: davanti al cancello nord del cimitero & p. t. s. \\
\hline 620 & Tonadico & $\begin{array}{l}\text { Ponte sul torrente Canali: a m } 2 \text { dall'estre- } \\
\text { mo destro del parapetto a monte }\end{array}$ & p. q. \\
\hline 621 & Selva di Cadore & $\begin{array}{l}\text { Ponte sul Rio Stretto: a destra del Rio, a } \\
\text { in } t, 7 \text { dal centro del ponte }\end{array}$ & p. q. \\
\hline 622 & Fusine di Zoldo & C. P.: a m 3,6 dalla porta & p. t. s. \\
\hline 623 & Cibiana di Cadore & $\begin{array}{l}\text { C. P.: a m } t, t \text { dalla parete nord ovest del } \\
\text { campanile }\end{array}$ & p. q. \\
\hline 624 & Borca di Cadore & S. F.: fra la stazione e il binario & M. \\
\hline 625 & Ospitale di Cadore & $\begin{array}{l}\text { S. F.: all inizio della scalinata che porta ai } \\
\text { binari }\end{array}$ & p. d. $f$ \\
\hline 626 & Agordo & $\begin{array}{l}\text { S. F.: sul piazzale di smistamento carro } \\
\text { merci }\end{array}$ & M. \\
\hline 627 & Agordo & $\begin{array}{l}\text { Cantoniera provinciale in località c. della } \\
\text { Pissa a km } 2 \text { a sud di Muda: davanti } \\
\text { alla facciata est }\end{array}$ & p. q. \\
\hline 628 & Servo & Chiesa di S. Rocco: a m 3,5 dalla porta & p. t. \\
\hline 629 & Arten & $\begin{array}{l}\text { Incrocio di strade campestri a } \mathrm{km} \text { l,5 da } \\
\text { Arten verso Primolano: sul ciglio destro } \\
\text { della statale. }\end{array}$ & p. q. \\
\hline 630 & Sottoguda & C. P.: a m 3 dalla porta principale & p. q. \\
\hline 631 & Passo Fedaia & $\begin{array}{l}\text { Rifugio Passo Fedaia: a m } 2,6 \text { dalla fac- } \\
\text { ciata sud }\end{array}$ & p. q. \\
\hline 632 & Sottoguda & $\begin{array}{l}\text { Ultimo ponte sul torrente Pettorina andan- } \\
\text { do da Sottoguda a Malga Ciapela: sulla } \\
\text { sinistra a } \mathrm{m} 7,5 \text { dall'estremo sinistro del } \\
\text { paraghiaia a valle }\end{array}$ & p. q. \\
\hline 633 & Alleghe & $\begin{array}{l}\text { C. P.: a } m \underline{2} \text { a nord dello spigolo } \mathrm{N} \text {. E. } \\
\text { della sacrestia }\end{array}$ & M. \\
\hline 634 & Belluno & S. F.: davanti alla porta centrale & M. \\
\hline 635 & Tolmezzo & S. F.: davanti alla porta centrale & M \\
\hline 636 & Lorenzago di Cad. & C. P.: a $m+$ dalla porta principale & p. t. \\
\hline 637 & Denno & $\begin{array}{l}\text { Fabbrica di ceramiche a sud est di Denno } \\
\text { sulla statale della Val di Non: accanto } \\
\text { alla parete } \mathrm{N} \text {. in allineamento con la } \mathrm{S} \text {. } \\
\text { della casa } \mathrm{n} .136\end{array}$ & p. q. \\
\hline
\end{tabular}




\begin{tabular}{|c|c|c|c|}
\hline N. & $\begin{array}{l}\text { Località } \\
\qquad 2\end{array}$ & $\begin{array}{c}\text { Monografic } \\
3\end{array}$ & $\begin{array}{l}\text { Riferimento } \\
\text { altimetrico } \\
4\end{array}$ \\
\hline 6.38 & Tilio & C. P.: a m 3 dalla portal del cimpanile & $M$ \\
\hline 634 & Cles & C. P.: a $\mathrm{m}$ 6 dialla porta della pirets sud & p. t. s. \\
\hline $6+11$ & Romeno & $\begin{array}{l}\text { C.: accinte allat porticinal della parecte } \\
\text { orest }\end{array}$ & p. t. s. \\
\hline $6+1$ & Appiano & $\begin{array}{l}\text { S. F.: a m } 2.2 \text { diallia secondia porta da sin- } \\
\text { strat, guardando }\end{array}$ & p. l. i. \\
\hline $6+2$ & Civalcse & $\begin{array}{l}\text { C. P.: a m } 3, \bar{j} \text { dall apertura centrale del } \\
\text { recinto }\end{array}$ & p. t. \\
\hline $6+3$ & Passo di Lavazze & Ritugio Bucancre: a $\mathrm{m} 6.5$ clalla portil sucl & p. ч. \\
\hline $6+t$ & Bronzolo & s. F.: a m 3 dalla porta centrale & p. d. i. \\
\hline $6+5$ & Niturno & $\begin{array}{l}\text { S. F.: a } \mathrm{m} \text { I dialla facciata esest in allineal- } \\
\text { mento con la sud }\end{array}$ & p. i. i. \\
\hline $6+6$ & Lilces & $\begin{array}{l}\text { S. F.: a m } 2 \text { dalla ficiciata ovest in allinea- } \\
\text { mento con la sud }\end{array}$ & p. I. f. \\
\hline $6+7$ & Silindro & s. F.: a m 2,6 diallia portal centrale & p. il. i. \\
\hline 648 & Spondignal Prato & $\begin{array}{l}\text { S. F.: a } m \text { t.6 dalla parece nord in allinca- } \\
\text { mento con lat est }\end{array}$ & is. $\mathrm{r}$. \\
\hline $6+4$ & Saltusio & $\begin{array}{l}\text { Ponte in legno sul T. Passirio a } \mathrm{m} 600 \text { a } \\
\text { nord alel paese: a } m 4,6 \text { dall'inizio alel } \\
\text { ponte }\end{array}$ & p. $\mathbf{u}$ \\
\hline 650 & 'Terlano & $\begin{array}{l}\text { S. F.: a m } 2 \text { dalla paretc } \mathrm{N} \text {. F. in allinea- } \\
\text { mento con la S. F. }\end{array}$ & p. d. i. \\
\hline 651 & Mezzana & 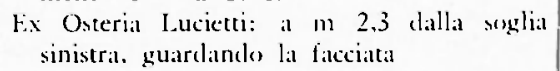 & $\therefore 1$. \\
\hline 652 & Jizzano & $\begin{array}{l}\text { Albergo Vittoria: a m } 5 \text { dalla parete N. O. } \\
\text { in allineamento con lia } \mathrm{N} \text {. E. }\end{array}$ & cs. $r$ \\
\hline 653 & Piazzola & Monumento ai Cacluti: a $m 2$ dal recinto & p. t. s. \\
\hline 654 & Mione & C. P.: a $m$ t.j dalla porta del campinile & p. t. s. \\
\hline 655 & Gomagoi & $\begin{array}{l}\text { Autorimessa Albergo Gallia: a } m 3 \text { dalla } \\
\text { parete est in allineamento con la sual }\end{array}$ & $\therefore \quad r$ \\
\hline 6.56 & Passo dello Stchio & $\begin{array}{l}\text { Albergo Passo dello Stelvio: a } m 2,7 \text { dal- } \\
\text { la parete nord in asse col finestrone cen- } \\
\text { trale }\end{array}$ & cs. $\therefore$ \\
\hline $65 \%$ & $\begin{array}{l}\text { Stracla dello Stcl. } \\
\text { vio }\end{array}$ & $\begin{array}{l}\text { Statale n. } 38 \text { dello Stelvio, Cantoniera n. 1: } \\
\text { a m } 3.2 \text { dal portone est }\end{array}$ & cs. $r$ \\
\hline 658 & Isolaccial & C. P.: all'inizio della stilinata & p. ч. \\
\hline 659 & $\begin{array}{l}\text { S. Antonio Mori- } \\
\text { gnone }\end{array}$ & $\begin{array}{l}\text { C. P.: a } m 2.5 \text { dalla parete sud in allinea- } \\
\text { mente con lal est }\end{array}$ & cs. $r$. \\
\hline 6611 & Riobianco & $\begin{array}{l}\text { Ponte sul Rio Bianco: sulla sinistra, a } \mathrm{m} 5 \\
\text { dallestremitat del parapetto a monte }\end{array}$ & p. ૫. \\
\hline 661 & Valclurna & C. P.: a m $2,+$ dalla porta del recinto & p. 4. \\
\hline 662 & Boscorival & $\begin{array}{l}\text { Ponte sul Rio Valdurna in località la Ce- } \\
\text { resira: a m } 15.5 \text { dial centro del ponte, sul- } \\
\text { la rival destra }\end{array}$ & p. 4. \\
\hline 663 & $\begin{array}{l}\text { S. Valcntino alla } \\
\text { Mutal }\end{array}$ & Chiencttal S. Cassiano: a m 2.5 dallat factiata & cs. $r$ \\
\hline $66+$ & $\begin{array}{l}\text { S Pancrazio d'Ll. } \\
\text { timo }\end{array}$ & C. P.: a m 9,5 dal gradino della perta & p. t. s. \\
\hline 665 & S. Nicolos d'Ultimos & C. P.: $\mathrm{m} 1$ dal cancello in legno del recinto & p. t. s. \\
\hline 666 & $\begin{array}{l}\text { Maklonnil di Sena- } \\
\text { les }\end{array}$ & C. P.: a m 6,3 dialla porta principale & p. t. s. \\
\hline
\end{tabular}




\begin{tabular}{|c|c|c|c|}
\hline$\therefore$ & $\begin{array}{l}\text { Località } \\
\qquad 2\end{array}$ & $\begin{array}{c}\text { Monografic } \\
3\end{array}$ & $\begin{array}{l}\text { Riferimento } \\
\text { altimetrico } \\
4\end{array}$ \\
\hline 667 & Passo del Giovo & $\begin{array}{l}\text { Chiesetta: a m } 2.9 \text { dal primo gratino dellat } \\
\text { scalinata est }\end{array}$ & is. $v$ \\
\hline 608 & Plan in Passiria & C. P.: a in 22,6 dalla porta principale & p. 4. \\
\hline$(1,9)$ & $\begin{array}{l}\text { S. Maria alla Fon- } \\
\text { derial }\end{array}$ & Chiesetta: a m 9,5 dalla soglia & p. 4 . \\
\hline 6,0 & Gianda & $\begin{array}{l}\text { Casia n. Ht: a } m 3 \text { dalla facciata } \mathrm{N} \text {. } \mathrm{E} \text {. in } \\
\text { allineamento con la } \mathrm{N} \text {. } O \text {. }\end{array}$ & p. 4 . \\
\hline 6,1 & Brunica & S. F.: a in 3 dalla porta principale & cs. $r$ \\
\hline 6,2 & Vipiteno & S. F.: a m 3,4 dalla porta principale & p. al. f. \\
\hline $6-3$ & Siletto & Chiesetta: a m 7,7 dalla porta est & p. t. s. \\
\hline $6-1$ & Mézzaselval & $\begin{array}{l}\text { Casello ferroviario 202-14t: a m } 1 \text { dalla } \\
\text { parete in corrispondenza del cs. }\end{array}$ & cs. $v$ \\
\hline 6,75 & Sciaves & $\begin{array}{l}\text { Casello ferroviario 4-780: a } m \text { dalla } \\
\text { parete in corrispondenza clel cs. }\end{array}$ & is. $v$ \\
\hline $6-6$ & S. Sigismondo & $\begin{array}{l}\text { Casello ferroviario 19-450: a } m \text { l, dalla } \\
\text { parete in corrispondenza del es. }\end{array}$ & is. $v$ \\
\hline 677 & Casteldarne & $\begin{array}{l}\text { S. F.: a m } 1.5 \text { lallia parete } S . O \text {. in alli- } \\
\text { neamento con la } N \text {. O. }\end{array}$ & cs. $Y$ \\
\hline $6-8$ & Gais & $\begin{array}{l}\text { C. P.: a m } 3.2 \text { dal cancelletto S. E. clel } \\
\text { recinto }\end{array}$ & p. t. s. \\
\hline $6,-9$ & Prato all'lsareo & $\begin{array}{l}\text { S. F.: a } m 1,3 \text { dal primo gradino della por- } \\
\text { ta principale }\end{array}$ & cs. $v$ \\
\hline 6,80 & Chiusa & $\begin{array}{l}\text { S. F.: a m } 0,9 \text { dal primo gradino della por- } \\
\text { ta principale }\end{array}$ & is. $r$ \\
\hline 6,81 & j'izzago & Chiesetta di S. Michele: a m 2.2 dalla porta & p. 1. \\
\hline $6 \times 2$ & luson & C. P.: a $\mathrm{m} 2$ dall'ingresso $\mathrm{S}$. E. del recinto & P. $\mathrm{q}$. \\
\hline 683 & S. Martino & $\begin{array}{l}\text { C. P.: a m } 2 \text { dalla parte N. O. del campa- } \\
\text { nile }\end{array}$ & p. i. \\
\hline $68+$ & Predoi & $\begin{array}{l}\text { C. P.: fra le due gradinate di accesso al sa- } \\
\text { grato, a } \mathrm{m} \text { l dal muro }\end{array}$ & p. t. s. \\
\hline 6,85 & Lappago & Cappella dAura: a $m$ l dalla porta & p. I. \\
\hline 686 & Monguelfo & S. F.: a $m 2,2$ dalla finestra centrale & is. $r$. \\
\hline $68 ;$ & Sesto & $\begin{array}{l}\text { C. P.: a } m+42 \text { dalla parete } N \text {. E. del cam- } \\
\text { panile }\end{array}$ & p. t. s. \\
\hline 688 & $\begin{array}{l}\text { S. Martino in Cia- } \\
\text { sies }\end{array}$ & C. P.: a m 3 dal cancelletto del recinto & p. t. s. \\
\hline $6 \times 4$ & Valdaora & $\begin{array}{l}\text { Albergo Stazione: a } m 4.5 \text { clalla facciata in } \\
\text { corrispondenza del cs. }\end{array}$ & is. $r$. \\
\hline 600 & Salornor & $\begin{array}{l}\text { S. F.: a m } 2 \text { dal primo gradino della porta } \\
\text { contrale }\end{array}$ & p. d. t. \\
\hline (19) & Riva di Tures & C. P.: a $\mathrm{m} 2$ dal cancelletto del recinto & p. t. s. \\
\hline 0,2 & Valduga & $\begin{array}{l}\text { Chiesetta: accanto al muretto del terrapieno } \\
\text { antistante lat chiesa in asse con la porta } \\
\text { principale }\end{array}$ & p. 4 \\
\hline 603 & Parrocchia (Chical) & C. P.: a m 5,6 dalla porta principale & J. t. s. \\
\hline 694 & $\operatorname{Tenno}$ & $\begin{array}{l}\text { Ristorante a Lago di Tenno w: a m l.t elial- } \\
\text { lat prima finestra destril guardinklo }\end{array}$ & p 1 . \\
\hline 6,95 & I.oppio & $\begin{array}{l}\text { C. P.: a m } 1.4 \text { alal primo graclino della } \\
\text { portia principale }\end{array}$ & $p 4$. \\
\hline 696 & $\begin{array}{l}\text { Castelletto di Bren- } \\
\text { zone }\end{array}$ & C. P.: davanti alla porta principale & P 1. \\
\hline
\end{tabular}




\begin{tabular}{|c|c|c|c|}
\hline N. & $\begin{array}{l}\text { Locilita } \\
\qquad 2\end{array}$ & $\begin{array}{c}\text { Monografiic } \\
\qquad 3\end{array}$ & $\begin{array}{l}\text { Riterimento } \\
\text { altimetrico } \\
4\end{array}$ \\
\hline 697 & Malcesine & $\begin{array}{l}\text { Municipio; a m } 0.5 \text { dalla porta dell'tificio } \\
\text { informazioni }\end{array}$ & cs. 0 . \\
\hline 698 & Arco & Municipio: a m 1,7 lal portone dentratil & cs. $v$. \\
\hline 699 & Dro & $\begin{array}{l}\text { C. P.: davanti alla porta laterale della fac- } \\
\text { ciata N. E. }\end{array}$ & cs. $v$ \\
\hline 700 & Vigo & C. P.: a m 2.7 alla porta principale & p. q. \\
\hline 701 & Vezzano & C. P.: davanti alla porti del campanile & cs. v. \\
\hline 702 & Borgo Valsugana & S. F.: davanti all'ingresso & p. d. f. \\
\hline 703 & Pontarso & $\begin{array}{l}\text { Ponte in legno sul } \mathrm{T} \text {. Rio Maso: sulla si- } \\
\text { nistra, a m } 13 \text { dal centro del ponte }\end{array}$ & p. ч. \\
\hline 704 & Cildonazzo & $\begin{array}{l}\text { Albergo Stazione: davanti alla porta d'in- } \\
\text { gresso }\end{array}$ & cs. v. \\
\hline 705 & Lavis & S. F.: a m 3,5 dalla porta centrale & p. d. f. \\
\hline 706 & Mosana & $\begin{array}{l}\text { Di fronte al primo capicello all'uscita del } \\
\text { paese per Verla, a destra della str.lda }\end{array}$ & p. q. \\
\hline 707 & Cembra & $\begin{array}{l}\text { Chiesal di S. Pictro: a m } 2,1 \text { dalla pirete } \\
\text { nord del campanile }\end{array}$ & p. t. s. \\
\hline 708 & Baselga di Pinè & Chiesa vecchia: a $m+$ dalla porta principale & p. t. \\
\hline 709 & Brusago & $\begin{array}{l}\text { In piazza: a } \mathrm{m} 2.6 \text { dalla buca delle lettere } \\
\text { posta alla sinistra del bar }\end{array}$ & p. q. \\
\hline 710 & S. Orsola & C. P.: davanti alla porta principale & p. q. \\
\hline 711 & Pergine & C. P.: a $m+$ dalla porta principale & is. 0 . \\
\hline 712 & Spiazzo & $\begin{array}{l}\text { C. P.: davanti alla porta sud in corrispon- } \\
\text { denza del caposaldo }\end{array}$ & cs. v. \\
\hline 713 & Molveno & $\begin{array}{l}\text { C. P.: a } \mathrm{m} 2,3 \text { dalla porticina del cimpal- } \\
\text { nile }\end{array}$ & p. t. s. \\
\hline 714 & Premione & C. P.: a m 2,4 dalla porta principale & p. t. \\
\hline 715 & $\begin{array}{l}\text { S. Giorgio di Co- } \\
\text { meglians }\end{array}$ & $\begin{array}{l}\text { C. P.: a m } 16 \text { dalla parete nord in allinea- } \\
\text { mento con la est }\end{array}$ & p. t. \\
\hline 716 & $\begin{array}{l}\text { S. Stefano di Ca- } \\
\text { dore }\end{array}$ & C. P.: a m 9,8 dalla porta principale & p. q. \\
\hline 717 & Val di Londo & $\begin{array}{l}\text { Al centro dello spazio tra i due ponti sul } \\
\text { Rio Cecido e sul Rin de Londo }\end{array}$ & p. q. \\
\hline 718 & Casera Pradibosco & $\begin{array}{l}\text { Colonia alpina di Pordenone: a m } 2 \text { dal- } \\
\text { l'ingresso della primia costruzione }\end{array}$ & p. $\mathbf{q}$. \\
\hline 719 & Pieria & C. P.: a $m 3,4$ dalla porta del campanile & p. t. s. \\
\hline 720 & Paularo & $\begin{array}{l}\text { C. P.: sulla strada, allinizio della rampa } \\
\text { che porta alla chiesa }\end{array}$ & p. t. s. \\
\hline 721 & Dordolla & $\begin{array}{l}\text { Strada Moggio-Pontebbal: in asse col ponte } \\
\text { in cemento sul ciglio ovest della strada }\end{array}$ & p. q. \\
\hline 722 & Cave del Predil & $\begin{array}{l}\text { Ponte sul Rio del Lago a } 200 \text { metri circa a } \\
\text { sud ovest della C. P.: a m } 7 \text { dall'estremo } \\
\text { ovest del parapetto a valle }\end{array}$ & p. q. \\
\hline 723 & Tamaroz & $\begin{array}{l}\text { Ponte di Tamaroz: a destra del Raccolana } \\
\text { a } \mathrm{m} 23,5 \text { dal pilone centrale }\end{array}$ & p. q. \\
\hline
\end{tabular}


Tabelat II - Valori osservati, coordinate geografiche, riduzioni ed anomalie.

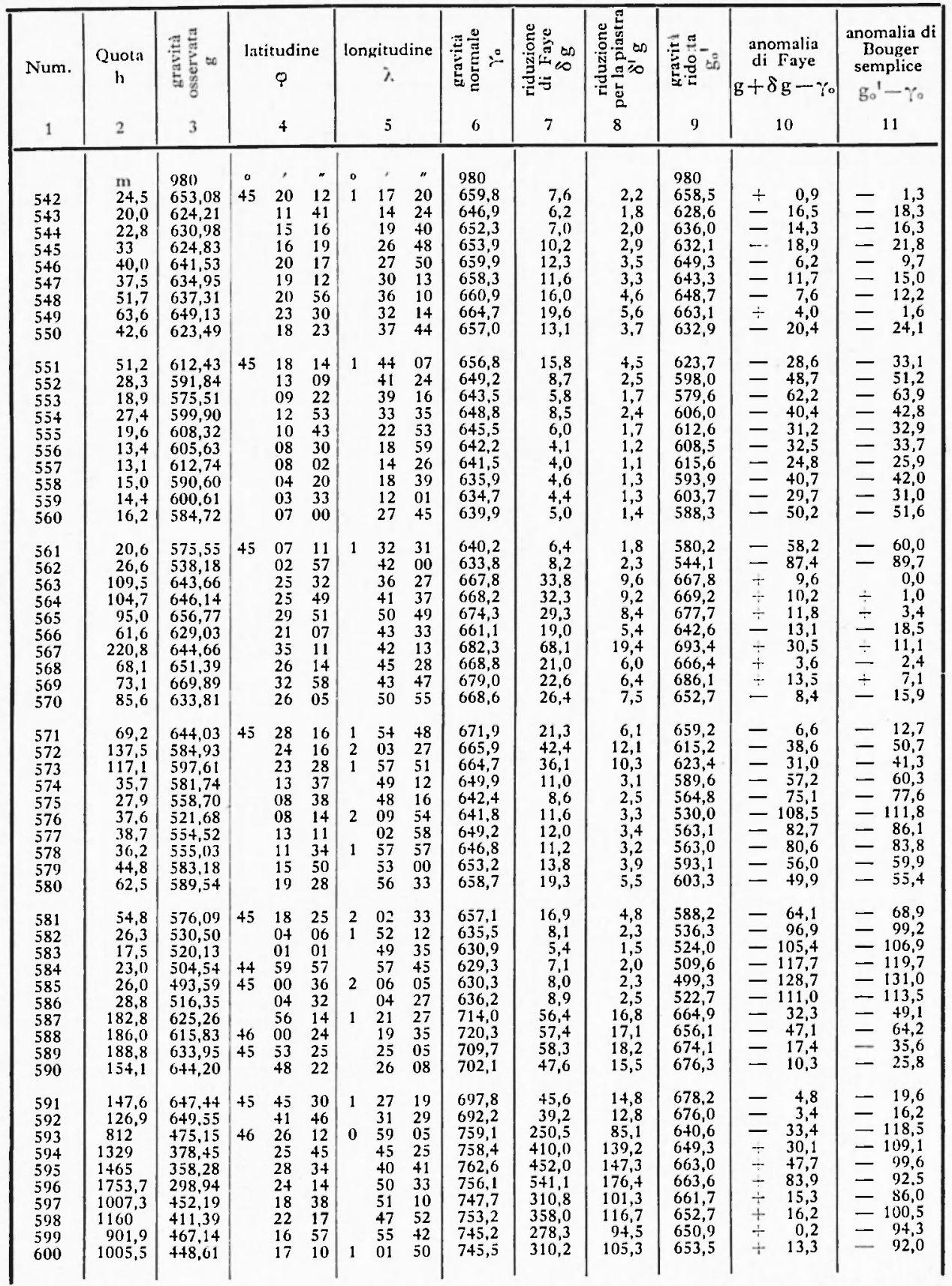




\begin{tabular}{|c|c|c|c|c|c|c|c|c|c|c|c|c|c|c|}
\hline Num. & $\begin{array}{c}\text { Quota } \\
\text { h } \\
2\end{array}$ & 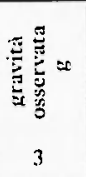 & & 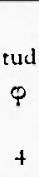 & & & $\begin{array}{r}\text { ngitu } \\
\lambda .\end{array}$ & & 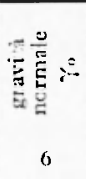 & 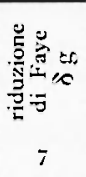 & 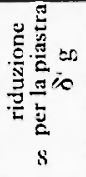 & 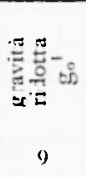 & $\begin{array}{c}\text { anomalia } \\
\mathrm{di} \text { Faye } \\
\mathrm{g}+\delta \mathrm{g}-\because \circ \\
10\end{array}$ & $\begin{array}{l}\text { anomalia di } \\
\text { Bouger } \\
\text { semplice } \\
\mathrm{s}^{-1}-\text { io } \\
11\end{array}$ \\
\hline & $\mathrm{m}$ & 980 & 0 & , & $\pi$ & o & , & $*$ & 980 & & & 980 & & \\
\hline 601 & 1007,3 & $+20,01$ & +6 & 18 & +0 & 1 & 03 & 34 & $7+7,7$ & 338.6 & $12+, 2$ & (034, 4 & 10,9 & $-113,3$ \\
\hline 602 & 1600,6 & 339,01 & & 29 & +7 & 0 & 34 & 42 & $76+.5$ & 403,9 & 161,0 & 671,0 & 08,4 & $-\quad 92,6$ \\
\hline 603 & $16+5$ & 333,25 & & 33 & 16 & & 35 & 51 & 769,7 & 507,6 & 165,4 & 675,4 & -71.2 & $-9+, 2$ \\
\hline 604 & 1591,5 & 339,10 & & 32 & 47 & & 40 & 58 & 769,0 & $+91,0$ & 160,1 & 070,0 & 61,1 & 99,0 \\
\hline 605 & 1223,9 & $+10,+9$ & & 34 & 32 & & +6 & 59 & 771,6 & 377,6 & 118,0 & 670,1 & 16,5 & $-101,5$ \\
\hline 606 & 773 & $+9,10$ & & 21 & 03 & & 29 & $0 \overline{5}$ & 751,3 & 238,5 & $7+, 5$ & $60,0.1$ & 16,7 & $-\quad 91,2$ \\
\hline 607 & 976,2 & +6121 & & 21 & 36 & & 32 & 13 & 752,1 & 301.2 & $9+1$ & 608,3 & 10,2 & 83,9 \\
\hline 608 & 2106 & $2+5,77$ & & 31 & 07 & & 20 & 36 & 766,4 & $\left(14^{9}, 8\right.$ & 220,6 & 675,0 & $-120,1$ & $-\quad 91.5$ \\
\hline 609 & 1224,8 & $+12,81$ & & 32 & 19 & & 18 & 54 & 768,3 & 377,9 & 128,3 & 662,4 & $-\quad 22,4$ & $-105,9$ \\
\hline 610 & $1+65$ & 368,86 & & 36 & 02 & & 18 & 51 & 773,8 & $+52,0$ & 153,5 & 667.4 & $=47,1$ & -106.4 \\
\hline 611 & 1121 & $+32,72$ & +6 & 31 & 56 & 0 & 09 & 48 & 767.7 & $3+5.9$ & 117,4 & 061,2 & 10.9 & $-106,5$ \\
\hline 612 & 969 & $46+, 72$ & & 34 & 30 & & 05 & 31 & 771,3 & 299,0 & 101,5 & 062,2 & 7,8 & $-109,3$ \\
\hline 613 & 1914 & 281,31 & & 22 & 40 & & 39 & 28 & 753,8 & 590,6 & 200,5 & 671,4 & $+\quad 118.1$ & $-\quad 82,4$ \\
\hline 614 & 1566 & 352,18 & & 18 & 32 & & +1 & +2 & 747.5 & +83.2 & $16+, 1$ & 671,3 & $-\quad 87.9$ & 76,2 \\
\hline $61 \overrightarrow{5}$ & $1+67$ & 374,67 & & 15 & +1 & & 38 & 56 & $7+3.3$ & $+52,7$ & 159,9 & 667,4 & $8+.0$ & 75,9 \\
\hline 616 & $1+07$ & $39+, 59$ & & 115 & 29 & & 48 & 33 & 727.9 & $+34,1$ & $1+1,5$ & 687,2 & -100.8 & $-\quad+10,7$ \\
\hline 617 & 1616 & 356,77 & & 07 & 06 & & +5 & 50 & 730,3 & $+98,6$ & $10^{0} 9,3$ & 680,1 & $-125,1$ & $-\quad 4+, 2$ \\
\hline 618 & 897,1 & +1) 1,60 & & 09 & 25 & & +2 & +4 & 733,8 & 276,8 & 97.8 & 670,6 & $\div 34,6$ & $-\quad 63.2$ \\
\hline 619 & 637 & $5+2,88$ & & 09 & 12 & & 38 & +1 & 733,5 & 196,5 & 66,7 & 672,7 & 5.9 & $-\quad 60,8$ \\
\hline 620 & 794 & 508,49 & & 11 & 07 & & 36 & 08 & 736,4 & $2+5,0$ & 86,5 & 607,0 & $\div \quad 17,1$ & $-69,4$ \\
\hline 621 & 1409 & 382,17 & 46 & 26 & 49 & 0 & 23 & 57 & 760,0 & 434.7 & 135,8 & 681,1 & 56,9 & 78,9 \\
\hline 622 & 1175,3 & $+33,01$ & & 22 & 24 & & 19 & 25 & $753 .+$ & 362,6 & 113,3 & 682,3 & $+2,2$ & 71,1 \\
\hline 623 & 1026 & $+68,70$ & & 23 & 26 & & 09 & 36 & $75+, 9$ & 316.6 & 107,5 & 677,8 & 30,4 & 77,1 \\
\hline 624 & 956,0 & $+62,94$ & & 26 & 05 & & 13 & 39 & 758,9 & 295,2 & 100,2 & 657,9 & 0,8 & $-101,0$ \\
\hline 625 & $+98,5$ & $56+, 58$ & & 19 & 31 & & 08 & 02 & $7+9,0$ & 153,8 & 50.1 & 668,3 & 30,0 & $-\quad 80,7$ \\
\hline 626 & $60+, 0$ & $5+6,60$ & & 16 & 52 & & 25 & 09 & $7+5,0$ & $186, t$ & 60,7 & 672,4 & 11,9 & $-\quad 72,6$ \\
\hline 627 & +56 & $56+, 73$ & & 13 & +2 & & 20 & 18 & $7+0,3$ & $1+0,7$ & 43,9 & 661,5 & 34,9 & 78,8 \\
\hline 628 & $66+, 7$ & $5+6,59$ & & 03 & 32 & & 40 & 22 & 725.0 & 205.1 & 69,6 & 682,1 & 20,7 & $+2,9$ \\
\hline 629 & 305 & 622,01 & & 00 & 14 & & 38 & 07 & 720.0 & 94,1 & 30,7 & 685,4 & 3,9 & 34,6 \\
\hline 630 & 1252 & 399,80 & & 25 & $2 S$ & & 30 & 50 & 758,0 & 386,3 & $1+1.7$ & $6+4,4$ & 28,1 & $-113,6$ \\
\hline 631 & 2056 & 250,78 & 46 & 27 & 11 & 0 & 33 & 52 & 760,6 & 634,4 & 232.7 & 652,5 & $\div \quad 124,6$ & -108.1 \\
\hline 632 & $1+04$ & 368,88 & & 25 & 32 & & 32 & 06 & 758,1 & $+33,2$ & 158,9 & $6+3,2$ & $-4+11$ & $-11+.9$ \\
\hline 633 & 978,8 & $+57,95$ & & 24 & 22 & & 25 & 55 & 756,3 & 302,0 & 98.4 & 061,6 & 3,7 & $-94,7$ \\
\hline 634 & 392,7 & 606.42 & & 08 & 26 & & 14 & 31 & 732.3 & 121.2 & 37.8 & 689,8 & 4,7 & +2.5 \\
\hline 635 & 315,1 & 617,31 & & 24 & 03 & & 34 & $02 \mathrm{E}$ & 755,8 & 97,2 & 30.4 & $68+.1$ & $+1,3$ & 71,7 \\
\hline 636 & 880,5 & 506,53 & & 28 & +6 & & 01 & $21 \mathrm{E}$ & 762.9 & 271.7 & 88,6 & 689.6 & 15,3 & 73,3 \\
\hline 637 & $299^{\circ}$ & 563,47 & & 15 & 15 & 1 & 23 & 18 & $7+2,6$ & () 2,3 & 30,1 & 625,7 & 86,8 & $-116,9$ \\
\hline 638 & 515 & 528,58 & & 19 & 14 & & 23 & 08 & 748,6 & 158.9 & +9.6 & 637,9 & 61,1 & $-110,7$ \\
\hline 639 & 658 & $+98,62$ & & 21 & $5 i$ & & 24 & 59 & 752,5 & 203,0 & 63.4 & 638,2 & 50,9 & $-11+, 3$ \\
\hline $6+0$ & 961 & $4+4,67$ & & 23 & 34 & & 19 & $5 \overline{5}$ & $75 \overline{5}, 1$ & 296,5 & 100.7 & $6+0.5$ & 13,9 & $-11+, 6$ \\
\hline $6+1$ & 399,5 & $5+3,20$ & 46 & 27 & 09 & 1 & 11 & 03 & 760,5 & 123,3 & $+1,9$ & $62+, 6$ & 94.0 & $-135,9$ \\
\hline $6+2$ & 903 & 451.63 & & 17 & 11 & & 59 & 24 & $7+5.5$ & 306.4 & 112,4 & $6+5,6$ & 12,5 & $-\quad 90,9$ \\
\hline $6+3$ & 1805 & 286,47 & & 21 & 15 & & 57 & 35 & 751.6 & 556,9 & 204.2 & $63^{\circ}, 2$ & 91,8 & $-112,4$ \\
\hline $6+4$ & 227,6 & 572.27 & & 24 & 16 & & 08 & 10 & 750,2 & 70,2 & 20,0 & 622.5 & $-113,7$ & $-133,7$ \\
\hline $6+5$ & 533,5 & 494,30 & & 38 & 39 & & 26 & 54 & 777.8 & 164,6 & 55,9 & 603,0 & $-118,9$ & $-174,8$ \\
\hline $6+6$ & 632.4 & $+55,51$ & & 37 & 09 & & 35 & 32 & 775.5 & 195,1 & 66.3 & $58+.3$ & $-124,9$ & $-191,2$ \\
\hline $6+7$ & $7+4,7$ & 430,36 & & 37 & 32 & & +1 & 10 & 776,1 & 229,8 & 78,0 & 582,2 & $-115,9$ & $\ldots 193,9$ \\
\hline $6+8$ & 885,2 & 399,23 & & 38 & 00 & & 50 & 29 & 776.8 & 273,1 & 92.7 & 579.6 & $-104,5$ & $-197,2$ \\
\hline $6+9$ & 476 & $504.3+$ & & $4+$ & 04 & & 15 & 10 & 785,9 & 146,9 & 53,9 & 597,3 & $-134,7$ & -188.6 \\
\hline 650 & 249,5 & 568,97 & & 31 & 40 & & 12 & 13 & 767.3 & 77,0 & 26,1 & 619,9 & -121.3 & $-1+7,4$ \\
\hline 651 & 899,8 & $+31,27$ & 46 & 18 & 59 & 1 & 38 & +6 & 748,2 & 277,6 & 101,8 & 607,1 & 39,3 & $-1+1,1$ \\
\hline 652 & $125+, 7$ & 359.04 & & 17 & 39 & & +5 & 56 & 746,2 & 387,2 & $1+2,0$ & $60+.2$ & 0,0 & $-1+2,0$ \\
\hline 653 & 1314 & $3+3,+7$ & & 24 & 36 & & 38 & 00 & 756,7 & 405,4 & 154,2 & $59+, 7$ & 7,8 & $-162,0$ \\
\hline $65+$ & 960 & $+33,83$ & & 26 & 06 & & 25 & 25 & 758,9 & 296,2 & 104,6 & 625.4 & 289 & $-133,5$ \\
\hline 655 & $12+1,8$ & 318,96 & & 34 & 37 & & 54 & 37 & 771,7 & 383,2 & $1+0,5$ & 561,7 & 69,5 & $-210,0$ \\
\hline 656 & 2757,4 & $05+, 76$ & & 31 & +1 & & 59 & 53 & 767.3 & 850,8 & 312.0 & 593,6 & $\therefore \quad 138,3$ & -173.7 \\
\hline 657 & 1718.4 & 236.04 & & 30 & 32 & 2 & 04 & +5 & 765,6 & 530,2 & 180,0 & 586,2 & 0,6 & $-179,4$ \\
\hline 65 & $13+5$ & 303,79 & & 29 & 17 & & 09 & 19 & 763,7 & $+15,0$ & 140,6 & 572,2 & $+4,9$ & $-191,5$ \\
\hline 659 & 1070,8 & $3+5,91$ & & 23 & 54 & & 05 & 50 & 755,6 & 330,4 & 130,1 & $5+6,2$ & 79,3 & $-200,4$ \\
\hline 660 & 1338 & 368,07 & & 46 & 17 & 1 & 05 & 01 & 789,3 & $+12,8$ & 151,4 & 629,5 & 8,4 & $-159,8$ \\
\hline
\end{tabular}




\begin{tabular}{|c|c|c|c|c|c|c|c|c|c|c|c|c|c|c|}
\hline $\begin{array}{c}\text { Num. } \\
1\end{array}$ & $\begin{array}{c}\text { Quota } \\
\text { h } \\
2\end{array}$ & 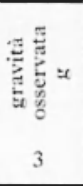 & & $\varphi$ & & $\begin{array}{r}\text { longitu } \\
\lambda\end{array}$ & dine & 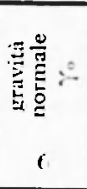 & 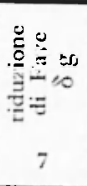 & 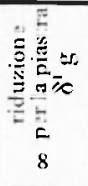 & $\begin{array}{c}\text { gravita } \\
\text { ridotta } \\
\mathrm{go}_{0}^{\prime} \\
9\end{array}$ & $\begin{array}{c}\text { ano } \\
\mathrm{di} \\
\mathrm{g} \div \delta\end{array}$ & $\begin{array}{c}\underset{\text { omalia }}{\text { Faye }} \\
\mathrm{g}-\because \circ \\
10\end{array}$ & 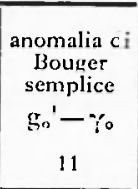 \\
\hline $\begin{array}{l}661 \\
662 \\
663 \\
664 \\
665 \\
666 \\
667 \\
668 \\
669 \\
670\end{array}$ & $\begin{array}{c}\mathrm{m} \\
1568 \\
1227 \\
1+54,2 \\
732,7 \\
1256 \\
1509 \\
1989,7 \\
1627 \\
1556 \\
1268\end{array}$ & $\begin{array}{l}980 \\
334,64 \\
39+, 34 \\
312,86 \\
465,64 \\
362,06 \\
301,27 \\
258,46 \\
307,32 \\
270,29 \\
329,69\end{array}$ & +6) & $\begin{array}{l}5 \\
44 \\
41 \\
45 \\
35 \\
30 \\
43 \\
50 \\
47 \\
31 \\
33\end{array}$ & $\begin{array}{l}20 \\
20 \\
16 \\
33 \\
10 \\
40 \\
19 \\
33 \\
42 \\
54 \\
16\end{array}$ & $\begin{array}{cc}0 \\
1 & 00 \\
02 \\
54 \\
21 \\
31 \\
35 \\
07 \\
21 \\
42 \\
40\end{array}$ & $\begin{array}{l}5 \\
49 \\
58 \\
58 \\
57 \\
43 \\
38 \\
30 \\
45 \\
16 \\
10\end{array}$ & $\begin{array}{l}980 \\
786,3 \\
781,7 \\
788,2 \\
772,5 \\
765,8 \\
784,8 \\
795,7 \\
791,4 \\
767,6 \\
769,7\end{array}$ & $\begin{array}{l}483,8 \\
378,6 \\
4+8,7 \\
226,1 \\
387,5 \\
465,6 \\
613,9 \\
502,0 \\
480,1 \\
391,2\end{array}$ & $\begin{array}{r}177,4 \\
138,8 \\
164,6 \\
82,9 \\
1+2,1 \\
170,8 \\
225,1 \\
190,9 \\
176,1 \\
1+3,5\end{array}$ & $\begin{array}{l}980 \\
6+1,0 \\
634,1 \\
597,0 \\
608,8 \\
607,5 \\
596,1 \\
647,3 \\
618,4 \\
574,3 \\
577,4\end{array}$ & $\begin{array}{l} \pm \\
= \\
= \\
\pm \\
=\end{array}$ & $\begin{array}{r}32,1 \\
8,8 \\
26,6 \\
80,8 \\
16,2 \\
17,9 \\
76,7 \\
17,9 \\
17,2 \\
48,8\end{array}$ & $\begin{aligned}= & 1+5,3 \\
= & 1+7,6 \\
= & 191,2 \\
= & 163,7 \\
= & 158,3 \\
= & 188,7 \\
= & 148,4 \\
= & 173,0 \\
= & 193,3 \\
- & 192,3\end{aligned}$ \\
\hline $\begin{array}{l}671 \\
672 \\
673 \\
674 \\
675 \\
676 \\
677 \\
678 \\
679 \\
680\end{array}$ & $\begin{array}{r}829,1 \\
948,3 \\
1381 \\
804,8 \\
756,8 \\
761,3 \\
786,8 \\
842 \\
315,8 \\
524,4\end{array}$ & $\begin{array}{l}+97,83 \\
+54,41 \\
366,99 \\
+62,95 \\
+99,95 \\
497,74 \\
501,47 \\
+80,41 \\
572,65 \\
5+1.18\end{array}$ & 46 & $\begin{array}{l}47 \\
53 \\
56 \\
48 \\
411 \\
48 \\
47 \\
49 \\
29 \\
38\end{array}$ & $\begin{array}{l}38 \\
+2 \\
54 \\
20 \\
16 \\
33 \\
49 \\
57 \\
4+ \\
32\end{array}$ & $\begin{array}{ll}0 & 31 \\
1 & 110 \\
0 & 54 \\
& 52 \\
& 47 \\
& 39 \\
& 36 \\
& 30 \\
1 & 00 \\
0 & 52\end{array}$ & $\begin{array}{l}19 \\
44 \\
47 \\
48 \\
34 \\
20 \\
30 \\
16 \\
22 \\
45\end{array}$ & $\begin{array}{l}791,3 \\
800,4 \\
805,2 \\
792,3 \\
789,2 \\
792,7 \\
791,6 \\
794,8 \\
764,4 \\
777,6\end{array}$ & $\begin{array}{r}255,8 \\
292,6 \\
+26,1 \\
2+8,3 \\
233,5 \\
23+, 9 \\
242,8 \\
259,8 \\
97,4 \\
161,8\end{array}$ & $\begin{array}{r}93,8 \\
107,3 \\
156,3 \\
91,1 \\
85,6 \\
86,1 \\
89,0 \\
95,3 \\
35,7 \\
59,3\end{array}$ & $\begin{array}{l}659,8 \\
639,7 \\
636,8 \\
620,2 \\
647,9 \\
646,5 \\
655,3 \\
64+, 9 \\
634,4 \\
6+3,7\end{array}$ & $\begin{array}{l}= \\
\bar{z} \\
\bar{z} \\
\bar{z} \\
\bar{z}\end{array}$ & $\begin{array}{l}37,7 \\
53,4 \\
12,1 \\
81,0 \\
55,7 \\
60,1 \\
47,3 \\
54,6 \\
94,3 \\
74,6\end{array}$ & $\begin{array}{r}131,5 \\
=160,7 \\
=168,4 \\
=172,1 \\
=1+1,3 \\
=146,2 \\
=136,3 \\
=149,9 \\
=130,0 \\
-133,9\end{array}$ \\
\hline $\begin{array}{l}681 \\
682 \\
683 \\
684 \\
685 \\
686 \\
687 \\
688 \\
689 \\
690\end{array}$ & $\begin{array}{l}1090 \\
970 \\
1002,0 \\
1+69 \\
1592 \\
1266 \\
1090,1 \\
1316 \\
1319 \\
1031,2\end{array}$ & $\begin{array}{l}+42,63 \\
+66,79 \\
+38,71 \\
360,39 \\
3+7,34 \\
392,63 \\
+40,59 \\
+08,42 \\
404,91 \\
+61,93\end{array}$ & $\begin{array}{l}47 \\
+6\end{array}$ & $\begin{array}{l}38 \\
44 \\
58 \\
02 \\
56 \\
53 \\
45 \\
42 \\
48 \\
40\end{array}$ & $\begin{array}{l}26 \\
+4 \\
26 \\
19 \\
+9 \\
+7 \\
07 \\
08 \\
+0 \\
05\end{array}$ & $\begin{array}{ll}0 & 46 \\
41 \\
& 31 \\
& 19 \\
& 23 \\
& 38 \\
& 20 \\
& 06 \\
& 13 \\
& 25\end{array}$ & $\begin{array}{l}02 \\
27 \\
12 \\
53 \\
25 \\
07 \\
+2 \\
07 \\
25 \\
19\end{array}$ & $\begin{array}{l}777,4 \\
786.9 \\
807,5 \\
813,3 \\
805,1 \\
800,5 \\
787,5 \\
783,0 \\
792,8 \\
788,9\end{array}$ & $\begin{array}{l}336,3 \\
299,3 \\
309,2 \\
+53,2 \\
+91,3 \\
390,6 \\
336,4 \\
406,1 \\
+07,0 \\
318,2\end{array}$ & $\begin{array}{l}123,3 \\
109,8 \\
113,4 \\
166,2 \\
180,2 \\
143,3 \\
123,3 \\
143,4 \\
149,3 \\
114,6\end{array}$ & $\begin{array}{l}655,6 \\
656,3 \\
634,5 \\
647,4 \\
658,4 \\
639,9 \\
662,7 \\
671,1 \\
662,6 \\
665,5\end{array}$ & $\begin{array}{l} \pm \\
\pm \\
\pm \\
\pm \\
\pm \\
\pm\end{array}$ & $\begin{array}{r}1,4 \\
20,8 \\
59,6 \\
0,3 \\
33,5 \\
17,3 \\
1,5 \\
31,5 \\
19,1 \\
8,9\end{array}$ & $\begin{array}{r}121,9 \\
=130,6 \\
=173,0 \\
=165,9 \\
=146,7 \\
=160,6 \\
=124,9 \\
=111,9 \\
=130,2 \\
=123,4\end{array}$ \\
\hline $\begin{array}{l}691 \\
692 \\
693 \\
694 \\
695 \\
696 \\
697 \\
698 \\
699 \\
700\end{array}$ & $\begin{array}{l}211,5 \\
655 \\
812 \\
600 \\
223 \\
64 \\
67,3 \\
91,1 \\
122,7 \\
611\end{array}$ & $\begin{array}{l}569,50 \\
5+3,51 \\
530,70 \\
530,09 \\
621,24 \\
664,91 \\
656,43 \\
635,61 \\
621,30 \\
541,84\end{array}$ & $\begin{array}{l}46 \\
+5\end{array}$ & $\begin{array}{l}14 \\
52 \\
46 \\
50 \\
51 \\
41 \\
45 \\
55 \\
57 \\
58\end{array}$ & $\begin{array}{l}+2 \\
30 \\
35 \\
08 \\
21 \\
14 \\
+9 \\
08 \\
39 \\
+1\end{array}$ & $\begin{array}{ll}1 & 14 \\
19 \\
19 \\
38 \\
31 \\
31 \\
42 \\
38 \\
33 \\
32 \\
28\end{array}$ & $\begin{array}{l}57 \\
23 \\
44 \\
26 \\
29 \\
06 \\
41 \\
59 \\
27 \\
17\end{array}$ & $\begin{array}{l}7+1,8 \\
708,5 \\
699,5 \\
713,8 \\
706,6 \\
691,4 \\
698,3 \\
712,3 \\
716,1 \\
717,7\end{array}$ & $\begin{array}{r}65,3 \\
202,1 \\
250,5 \\
185,1 \\
68,8 \\
19,7 \\
20,8 \\
28.1 \\
37,9 \\
188.5\end{array}$ & $\begin{array}{r}18,6 \\
68,6 \\
85,1 \\
62,7 \\
23,4 \\
6,2 \\
6,5 \\
9,2 \\
12,3 \\
6+, 0\end{array}$ & $\begin{array}{l}616,2 \\
677,0 \\
696,2 \\
652,5 \\
666,6 \\
678,4 \\
670,7 \\
654,5 \\
646,9 \\
666,3\end{array}$ & $\begin{array}{l}\bar{z} \\
\bar{z} \\
\bar{z} \\
\bar{z} \\
\overline{\div}\end{array}$ & $\begin{array}{r}107,0 \\
37,1 \\
81,8 \\
1,4 \\
16,6 \\
6,8 \\
21,1 \\
48,6 \\
56,9 \\
12,6\end{array}$ & $\begin{array}{lr}= & 125,6 \\
= & 31,5 \\
= & 3,3 \\
= & 61,3 \\
= & 40,0 \\
= & 13,0 \\
= & 27,6 \\
= & 57,8 \\
= & 69,2 \\
- & 51,4\end{array}$ \\
\hline $\begin{array}{l}701 \\
702 \\
703 \\
704 \\
705 \\
700 \\
707 \\
708 \\
709 \\
710\end{array}$ & $\begin{array}{c}385,5 \\
390,1 \\
922 \\
468,4 \\
206,4 \\
+23 \\
665 \\
964,2 \\
1103 \\
925\end{array}$ & $\begin{array}{l}563,75 \\
578.91 \\
+57,64 \\
571,87 \\
588,34 \\
551,71 \\
505,60 \\
+58,60 \\
+22,24 \\
+65,99\end{array}$ & $\begin{array}{l}45 \\
46\end{array}$ & $\begin{array}{l}04 \\
03 \\
07 \\
59 \\
98 \\
09 \\
10 \\
07 \\
10 \\
06\end{array}$ & \begin{tabular}{l|l}
40 & \\
02 & 1 \\
06 & 1 \\
46 & \\
50 \\
09 \\
28 \\
56 \\
54 \\
22
\end{tabular} & $\begin{array}{ll}1 & 27 \\
0 & 59 \\
0 & 57 \\
1 & 11 \\
& 21 \\
& 18 \\
& 13 \\
& 12 \\
& 07 \\
& 08\end{array}$ & $\begin{array}{l}011 \\
53 \\
45 \\
13 \\
21 \\
45 \\
59 \\
33 \\
35 \\
57\end{array}$ & $\begin{array}{l}726,7 \\
724,2 \\
730,3 \\
719,3 \\
732,9 \\
733,4 \\
735,4 \\
731,6 \\
736,1 \\
729,2\end{array}$ & $\begin{array}{r}118,9 \\
120,4 \\
284,5 \\
1+4,5 \\
63,7 \\
130,5 \\
205,2 \\
297,5 \\
3+0,3 \\
285,4\end{array}$ & $\begin{array}{r}37,2 \\
+2,5 \\
104,3 \\
51,0 \\
19,0 \\
42,5 \\
72,5 \\
109,1 \\
124,8 \\
104,7\end{array}$ & $\begin{array}{l}6+5,5 \\
656,8 \\
637,8 \\
665,4 \\
633,0 \\
639,7 \\
638,3 \\
647,0 \\
637,7 \\
6+6,7\end{array}$ & $\begin{array}{l}E \\
- \\
- \\
= \\
\div \\
\div\end{array}$ & $\begin{array}{r}+4,0 \\
24,9 \\
11,8 \\
2,9 \\
80,9 \\
51,2 \\
24,6 \\
24,5 \\
26,4 \\
22,2\end{array}$ & $\begin{array}{l}-81,2 \\
=\quad 67,4 \\
=\quad 92,5 \\
-\quad 53,9 \\
=\quad 99,9 \\
=\quad 93,7 \\
=\quad 97,1 \\
-\quad 84,6 \\
-\quad 98,4 \\
-\quad 82,5\end{array}$ \\
\hline $\begin{array}{l}711 \\
712 \\
713 \\
714 \\
715 \\
716 \\
717 \\
718 \\
719 \\
720\end{array}$ & $\begin{array}{c}480,4 \\
651,3 \\
850,8 \\
607,7 \\
640,0 \\
908 \\
13+5 \\
1187 \\
674 \\
667\end{array}$ & $\begin{array}{l}557,90 \\
+86,38 \\
461,02 \\
522,14 \\
551,27 \\
495,81 \\
+15,55 \\
+43,11 \\
5+0,80 \\
5+9,21\end{array}$ & 46 & $\begin{array}{l}03 \\
06 \\
08 \\
02 \\
31 \\
33 \\
37 \\
30 \\
31 \\
31\end{array}$ & $\begin{array}{l}46 \\
06 \\
29 \\
51 \\
06 \\
27 \\
57 \\
39 \\
11 \\
47\end{array}$ & $\begin{array}{ll}1 & 12 \\
& 42 \\
& 29 \\
& 34 \\
0 & 24 \\
0 & 05 \\
& 09 \\
& 09 \\
& 13 \\
& 20 \\
& 39\end{array}$ & $\begin{array}{l}37 \\
48 \\
07 \\
39 \\
+9 \\
52 \\
57 \\
57 \\
25 \\
42 \\
42 \\
42\end{array}$ & $\begin{array}{l}725,3 \\
728,8 \\
732,4 \\
724,0 \\
766,4 \\
770,0 \\
776,7 \\
765,7 \\
766,5 \\
767,4\end{array}$ & $\begin{array}{l}148,2 \\
201,0 \\
265,3 \\
187,5 \\
197,5 \\
280,2 \\
+15,0 \\
366,2 \\
208,0 \\
205,8\end{array}$ & $\begin{array}{r}52,3 \\
73,7 \\
90,1 \\
61,1 \\
61,7 \\
95,1 \\
135,3 \\
114,4 \\
65,0 \\
67,1\end{array}$ & $\begin{array}{l}653,8 \\
613,7 \\
636,2 \\
648,5 \\
687,1 \\
680,9 \\
695,3 \\
694,9 \\
683,8 \\
687,9\end{array}$ & $\begin{array}{l}= \\
= \\
\bar{E} \\
+ \\
\pm \\
\overline{-}\end{array}$ & $\begin{array}{r}19,2 \\
41,4 \\
6,1 \\
14,4 \\
17,6 \\
6,0 \\
53,9 \\
43,6 \\
17,7 \\
12,4\end{array}$ & $\begin{array}{rr} & 71,5 \\
= & 115,1 \\
=\quad 96,2 \\
=\quad 75,5 \\
=\quad 79,3 \\
=\quad 89,1 \\
=\quad 81,4 \\
=\quad 80,8 \\
=\quad 82,7 \\
-\quad 79,5\end{array}$ \\
\hline
\end{tabular}




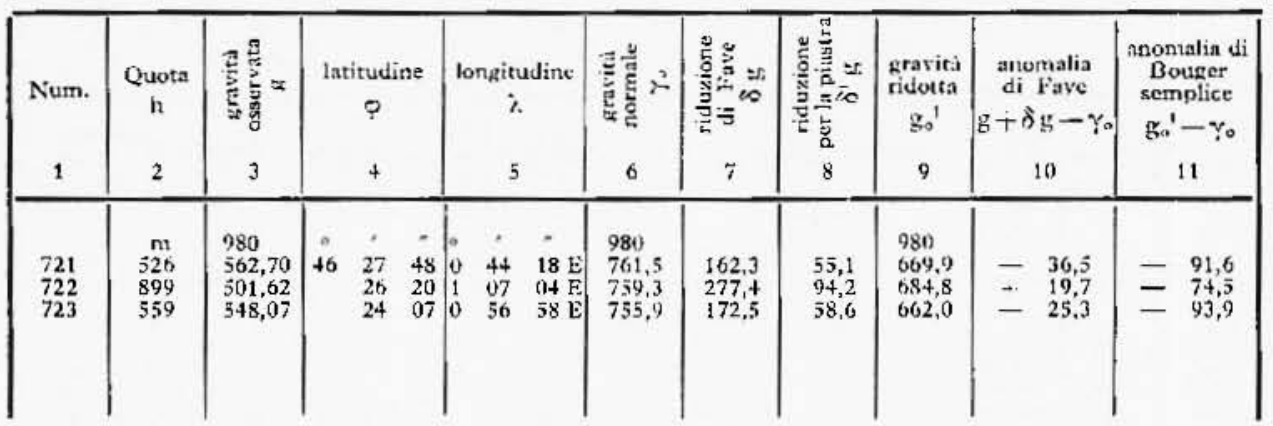




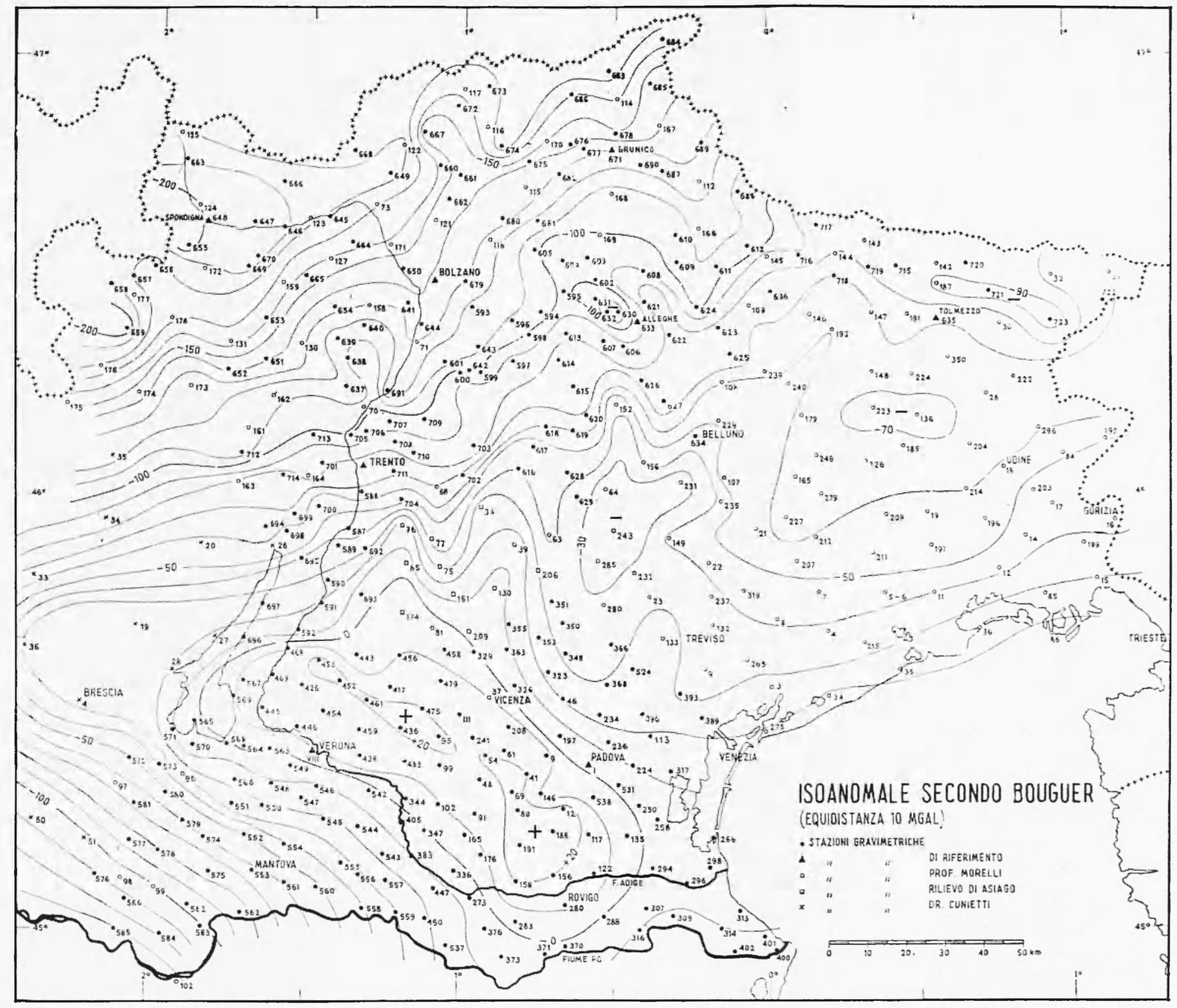

